\title{
A study of periprosthetic fractures following hemi-arthroplasty of hip in elderly population: diagnosis and management
}

\section{Gagandeep Mahi, Milind Atmaram Lokhande, Lokesh Kumar Yogi*, Ankit Gawri}

Department of Orthopaedics, GMCH, Aurangabad, Maharashtra, India

Received: 03 July 2020

Revised: 18 July 2020

Accepted: 23 July 2020

\section{*Correspondence:}

Dr. Lokesh Kumar Yogi,

E-mail: lokesh.k.yogi@gmail.com

Copyright: (c) the author(s), publisher and licensee Medip Academy. This is an open-access article distributed under the terms of the Creative Commons Attribution Non-Commercial License, which permits unrestricted non-commercial use, distribution, and reproduction in any medium, provided the original work is properly cited.

\begin{abstract}
Background: Hemi-arthroplasty of hip for intracapsular fractures of femoral neck is one of the most commonly done surgical procedures in the low mobility older Indian population. Although there are numerous complications associated with the procedure, we want to study the types and management of peri-prosthetic fractures in patients treated with hip hemi-arthroplasty.

Methods: A total of 26 patients were included in the study. A retrospective analysis of the radiological cum clinical diagnosis and management of peri-prosthetic fractures was performed. All patients were followed up till 1.5 years postoperatively.

Results: According to Vancouver classification of peri-prosthetic fractures, 10 fractures were classified as type A, 7 as type B1, 3 as type B2, 4 as type B3 and 2 as type C. Fractures were managed either by Open Reduction with Internal Fixation (ORIF) or Revision hemi-arthroplasty. Fractures type AG, B1 and C were managed with ORIF and type B2 and B3 fractures were managed with revision hemi-arthroplasty using longer stem.

Conclusions: As evident in our study, majority of fractures occurred intra-operatively, a thorough evaluation of preoperative x-rays of both hips should be done along with special focus on anatomical variations. Correct diagnosis based on radiological and intraoperative findings followed by appropriate treatment are of paramount importance in the management of peri-prosthetic fractures.
\end{abstract}

Keywords: Peri-prosthetic fractures, Revision surgery, Vancouver classification

\section{INTRODUCTION}

In elderly hip fractures pose a major public health problem. ${ }^{1}$ These fractures form around one-fourth of all fractures in patients aged 75 years and over. ${ }^{2}$ Femoral neck fractures occur most frequently in elderly female patients following fall from standing height and may be associated with osteoporosis. ${ }^{3}$ Hip hemi-arthroplasty is one of the recommended treatment options in the elderly low mobility population as the large diameter hemiarthroplasty head component reduces the risk of dislocation: total hip replacement is the recommended option in the more active population as it can provide a better functional outcome. ${ }^{4-9}$

Various complications like deep soft tissue infection, deep vein thrombosis, dislocation or implant failure can occur following hemi-arthroplasty of hip. Review of the three large scale studies, which reported on implant-related complications, revealed the most common complication was peri-prosthetic femoral fracture. ${ }^{10}$ Femoral periprosthetic fractures can occur during the index hemiarthroplasty surgery or postoperatively. The well-known Vancouver classification system, developed by Duncan and Masri et al for fractures of femur after total hip 
arthroplasty is considered the most reliable method for assessment of intra-/postoperative femoral fractures according to the location of the fracture and the presence of a well-fixed or loose component. ${ }^{11}$ This system is commonly used worldwide, and shows good correlation between radiological and intraoperative clinical findings. ${ }^{12,13}$ This classification system has also been altered to include intraoperative fractures and perforations. $^{14}$

The aim of this retrospective study is to analyze the diagnosis of peri-prosthetic fractures and their subsequent management in elderly patients who had undergone hemiarthroplasty of hip for fracture of femoral neck.

\section{METHODS}

A total of 26 patients from March 2018 to September 2019 who suffered from peri-prosthetic fractures during or after the uncemented hemi-arthroplasty of hip, in Government Medical College and Hospital, Aurangabad were included in the study. A retrospective analysis of the radiological and clinical diagnosis and management of peri-prosthetic fractures was performed. 17 out of 26 fractures were iatrogenic and occurred during the index surgery. 9 fractures were caused by low energy fall.

We assessed patient reports focusing on type of fracture, procedure done for management of said peri-prosthetic fracture, operative time and type of fixation. We used Vancouver classification system (Figure 1 and 2).

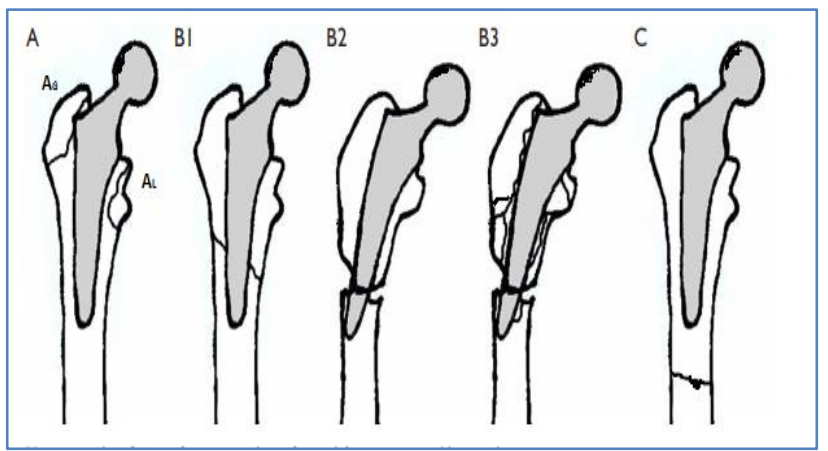

Figure 1: Vancouver classification for peri-prosthetic femoral fractures around hip implants. ${ }^{14}$

\section{Type A}

Involve proximal metaphysis (subdivided into AG or AL with greater trochanter and lesser trochanter involvement respectively).

\section{Type B}

Fractures occur around the stem or just below it (subdivided in B1 if the stem was stable, B2 in case of loose stem with adequate bone stock and B3 loose stem without fair bone stock).

\section{Type C}

Fractures occur below the stem tip.

Particular attention was paid on stem stability evaluation conducted in all patients with X-rays and intraoperative assessment. After the exposure the fracture site we accurately evaluated the stem stability for ultimate surgical decision.
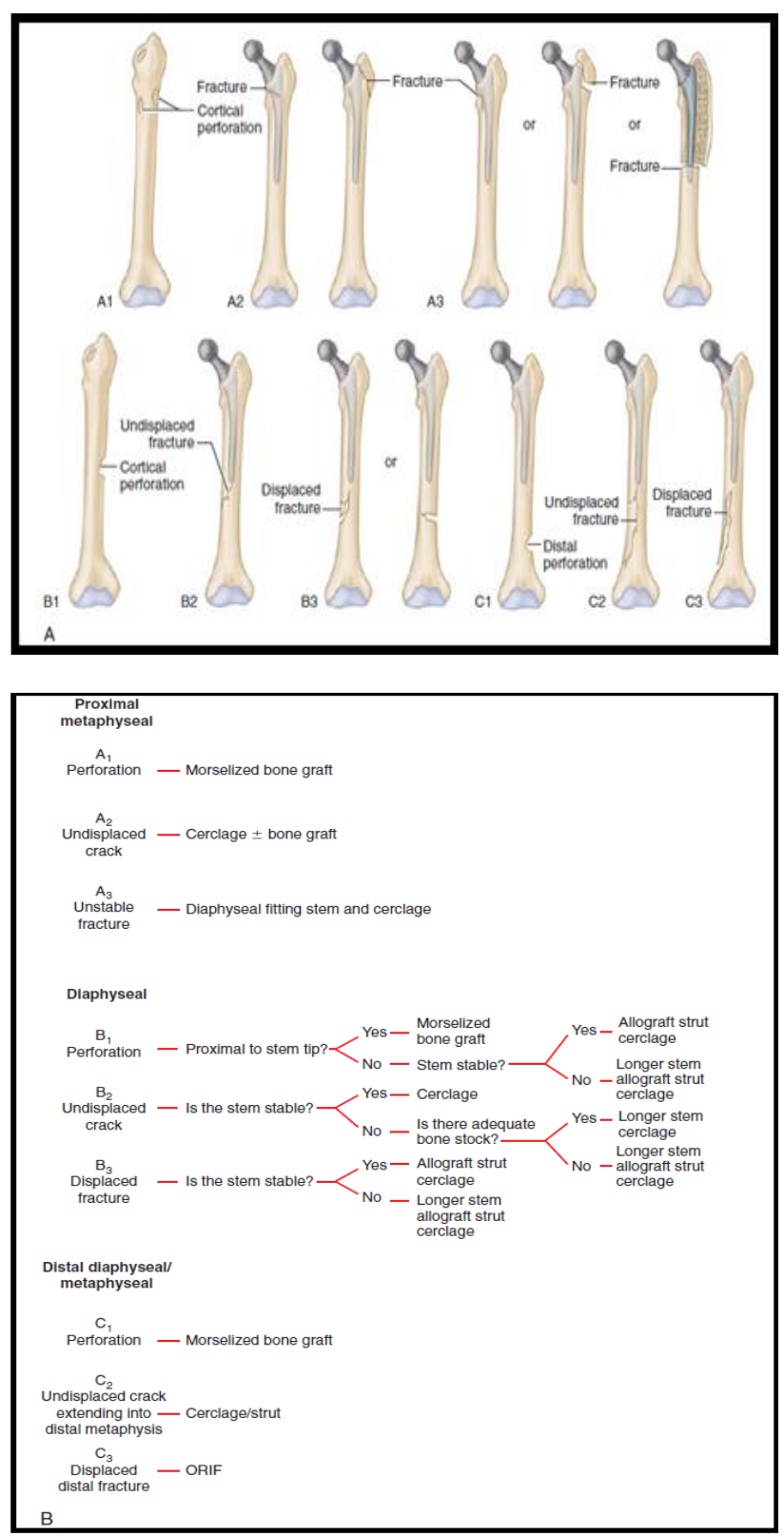

Figure 2: (A) Classification and management protocol and (B) intra-operative periprosthetic fractures of femur.

Redrawn from Greidanus, Mitchell, and Masri et al principles of management and results of treating the fractured femur during and after total hip arthroplasty, Instr Course Lect 52:309, 2003. ${ }^{14}$ 
All surgeries for peri-prosthetic fractures were performed by utilization of previously placed incision scar via posterior approach with proximal and distal extensions as and when needed. All surgeries for peri-prosthetic fractures were performed by utilization of previously placed incision scar via posterior approach with proximal and distal extensions as and when needed.

The treatment given was morselized bone graft obtained from extracted femoral head with or without cerclage (utilizing $\mathrm{K}$ wires and tension band wiring technique for AG fractures (Figure 3) for all type A fractures as they were recognized intra-operatively. Open reduction and internal fixation (ORIF) with original implant in-situ for all $\mathrm{B}_{1}$ (Figure 4) and all $\mathrm{C}$ fractures were done as the second and definitive procedure after the confirmation of diagnosis. Revision along with ORIF was required for all $\mathrm{B}_{2}$ and $\mathrm{B}_{3}$ cases. The length of hospitalization and time to union were analysed.

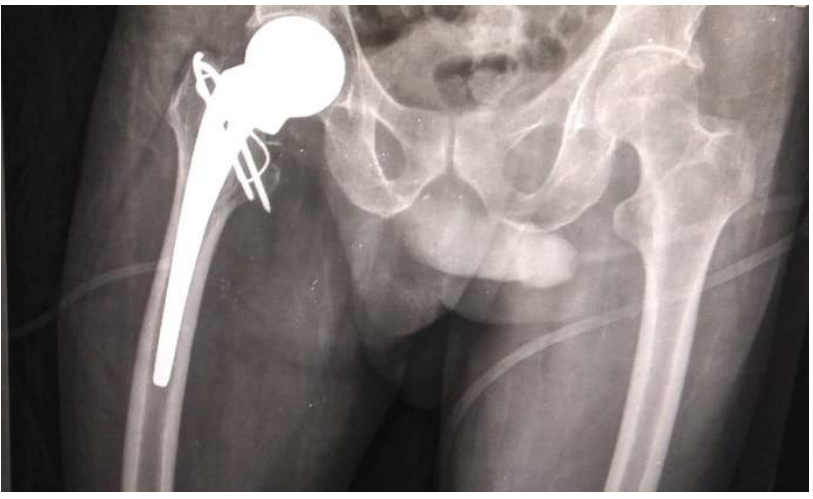

Figure 3: A case of type A Vancouver that occurred intra-operatively was managed by $T B W$ with $K$ wires.

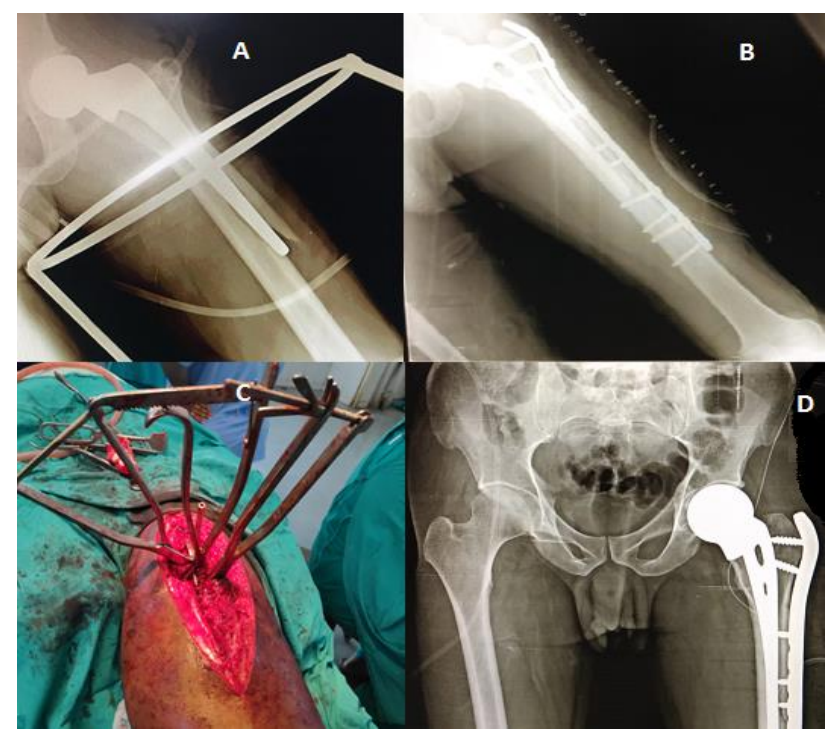

Figure 4 (A-D): A case of Vancouver B1 type periprosthetic fracture that occurred during insertion of bipolar prosthesis. Implant was revised to a smaller diameter unipolar AMP and ORIF was done with proximal femur locking plate.
All patients were followed up till 1.5 years postoperatively. Radiological and clinical evaluations were conducted after 1, 3, 6, 12 months and 18 months after the surgery for peri-prosthetic fractures.

Statistical analysis was done on microsoft excel sheet using SPSS25 utilizing microsoft excel 10 and microsoft word 10.

\section{RESULTS}

In the study 12 men and 14 women with a mean age of $73.38 \pm 8.01$ years at the time of presentation were included. Primary implant was unipolar AMP in 10 and bipolar prosthesis in 16 patients. According to Vancouver classification of peri-prosthetic fractures, 10 fractures were classified as type $A, 7$ as type $B_{1}, 3$ as type $B_{2}, 4$ as type $\mathrm{B}_{3}$ and 2 type $\mathrm{C}$ (Table 1 ).

Follow up was divided on the basis of the surgery done in type A fractures, fractures of greater trochanter (AG) were managed with tension-band wiring using $\mathrm{K}$ wires, fractures of lesser trochanter (AL) were managed using morselized local graft obtained from extracted head. All type $\mathrm{A}$ fractures healed uneventfully. Type $\mathrm{B}_{1}$ and $\mathrm{C}$ fractures were managed with ORIF (plate and screws); type $B_{2}$ and $B_{3}$ fractures were managed with a longer stem revision hemi-arthroplasty. The mean surgical time for operative procedure required for the treatment of periprosthetic fractures was $120 \pm 10$ minutes. The mean hospital stay of patients was 8 days. 22 out of 26 patients went on have uneventful union and reported no complications after the surgery and 4 patients developed infected non-union, 2 patients from type B2 with 1 from $\mathrm{B} 3$ and type $\mathrm{C}$ each.

Table 1: Demographic data of study $(n=26)$.

\begin{tabular}{|ll|}
\hline Variables & Values \\
\hline Sex & 14 \\
\hline Female & 12 \\
\hline Male & $73.38 \pm 8.01$ \\
\hline Mean age (in years) & \\
\hline Vancouver classification & 10 \\
\hline Type A & 7 \\
\hline Type $B_{1}$ & 3 \\
\hline Type $B_{2}$ & 4 \\
\hline Type $B_{3}$ & 2 \\
\hline Type C & $120 \pm 10$ \\
\hline Mean operative time (in minutes) & 8 days \\
\hline Mean hospital stay & 1.5 years \\
\hline Minimum follow up & \\
\hline Outcome & 22 \\
\hline Union & 4 \\
\hline Non-union & 2 \\
\hline Type B3 & 1 \\
\hline Type B2 & 1 \\
\hline Type C & \\
\hline
\end{tabular}




\section{DISCUSSION}

The prime goal of management of peri-prosthetic hip fracture is timely and correct diagnosis according to Vancouver classification, appropriate surgical procedure which ultimately leads to adequate bone healing and early return to pre-injury function. Advanced age, osteoporosis, co-morbidity and age-related weakness that lead to low energy trauma are cited as the most frequent cause of periprosthetic injuries. $^{15}$

In Lindhall et al study $47 \%$ of $\mathrm{B}_{2}$ fractures were classified initially as B1, in relation with radiological findings. ${ }^{16}$ Also Fleischman et al reported high number of failures in $\mathrm{B}_{1}$ fracture (assumed fix stem) treated with ORIF techniques comparing with $\mathrm{B}_{2}$ fracture underwent revision. ${ }^{17}$ Khan et al suggest accurate radiological evaluation especially in patients with pre-existing thigh pain. ${ }^{18}$ Specific localization of the fracture, such as near area of lysis below an apparently well fixed stem should lead to decide for revision surgery. ${ }^{19,20}$ This is supported by Swedish National Hip Arthroplasty Register data that show higher percentage of re-operation after ORIF without stem revision $32 \%$ compare with revision alone $10 \%$ or revision in association with internal fixation $23 \% .^{16}$

Although various standard head sizes ranging from 37 to 53 were available, due to economic constraints a universal implant type (either unipolar Austin Moore-Prosthesis or bipolar prosthesis) with fixed stem morphology was the only available implant that could be used routinely in a tertiary government center like ours. This may be associated with high rate of intra-operative peri-prosthetic fractures, especially type A variants. An appropriate surgical technique and an anticipation of narrow canal should warrant more gentle and diligently done procedure in these elderly patients with a weak bone stock.

\section{CONCLUSION}

As evident in our study, majority of fractures occurred intra-operatively, a thorough evaluation of pre-operative $\mathrm{X}$-rays of pelvis with both hips in both AP and lateral view should be done along with special focus on anatomical variations like excessive proximal femoral bowing. Correct diagnosis based on radiological and intraoperative findings followed by appropriate treatment are of paramount importance in the management of periprosthetic fractures. Although unipolar implants were associated with lesser frequency and severity of periprosthetic fractures, a larger study group is required for establishing that unipolar implants are better than bipolar implants for hemi-arthroplasty of hip for neck femur fractures.

\section{ACKNOWLEDGEMENTS}

We want to extend our token of appreciation towards our Professor and Head of the Department Dr. Chandrakant R. Thorat sir who has relentlessly worked for the betterment of our department and thank him for his everlasting support in all our endeavours.

Funding: No funding sources

Conflict of interest: None declared

Ethical approval: The study was approved by the institutional ethics committee

\section{REFERENCES}

1. Cooper C, Campion G, Melton LJ. Hip fractures in the elderly: a world-wide projection. Osteoporos Int. 1992;2:285-9.

2. Burge R, Hughes DB, Solomon DH, Wong JB, King A, Tosteson A. Incidence and economic burden of osteoporosis-related fractures in the United States, 2005-2025. J Bone Miner Res. 2007;22:465-47.

3. Saberi S, Arabzadeh A, Khomeisi B, Berehnegard E, Mortazavi SMJ. Early Complications Following Bipolar Hemiarthroplasty for Femoral Neck Fracture in Elderly Patients. Acad J Surg. 2014;1:3-4.

4. Hopley C, Stengel D, Ekkernkamp A, Wich M. Primary total hip arthroplasty versus hemiarthroplasty for displaced intracapsular hip fractures in older patients: systematic review. BMJ. 2010;340:c2332.

5. Wang F, Zhang H, Zhang Z, Ma C, Feng X. Comparison of bipolar hemiarthroplasty and total hip arthroplasty for displaced femoral neck fractures in the healthy elderly: a meta-analysis. BMC Musculoskelet Disord. 2015;16:229.

6. Carroll C, Stevenson M, Scope A, Evans P, Buckley S. Hemiarthroplasty and total hip arthroplasty for treating primary intracapsular fracture of the hip: a systematic review and cost-effectiveness analysis. Health Technol Assess. 2011;15:1-74.

7. Burgers PT, Geene VAR, Bekerom VMP, Lieshout VEM, Blom B, Aleem IS, et al. Total hip arthroplasty versus hemiarthroplasty for displaced femoral neck fractures in the healthy elderly: a meta-analysis and systematic review of randomized trials. Int Orthop. 2012;36:1549-60.

8. Goh SK, Samuel M, Su DH, Chan ES, Yeo SJ. Metaanalysis comparing total hip arthroplasty with hemiarthroplasty in the treatment of displaced neck of femur fracture. J Arthroplasty. 2009;24:400-6.

9. Zhao Y, Fu D, Chen K, Li G, Cai Z, Shi Y, et al. Outcome of hemiarthroplasty and total hip replacement for active elderly patients with displaced femoral neck fractures: a meta-analysis of 8 randomized clinical trials. PLoS One. 2014;9:e98071.

10. Veldman HD, Heyligers IC, Grimm B, Boymans TA. Cemented versus cementless hemiarthroplasty for a displaced fracture of the femoral neck: a systematic review and meta-analysis of current generation hip stems. Bone Joint J. 2017;99:421-31.

11. Duncan CP, Masri BA. Fractures of the femur after hip replacement. Instr Course Lect. 1995;44:293304. 
12. Brady OH, Garbuz DS, Masri BA, Duncan CP. The reliability and validity of the Vancouver classification of femoral fractures after hip replacement. J Arthroplasty. 2000;15(1):59-62.

13. Rayan F, Dodd M, Haddad FS. European validation of the Vancouver classification of periprosthetic proximal femoral fractures. J Bone Joint Surg Br. 2008;90(12):1576-9.

14. Frederick MA, Beaty JH, Canale ST. Campbell's Operative Orthopaedics. 4 volume set, 13th ed. Philadelphia: Elsevier; 2017: 255-256.

15. Giaretta S, Momoli A, Porcelli G, Micheloni GM. Diagnosis and management of periprosthetic femoral fractures after hip arthroplasty. Injury. 2019;50(2):29-33.

16. Lindahl H, Garellick $G$, Regner $H$, Herberts $P$, Malchau H. Three hundred and twenty-one periprosthetic femoral fractures. J Bone Joint Surg Am. 2006;88(6):1215-22.
17. Fleischman AN, Chen AF. Periprosthetic fractures around the femoral stem: overcoming challenges and avoiding pitfalls. Ann Transl Med. 2015;3(16):234.

18. Khan T, Grindlay D, Ollivere BJ, Scammell BE, Manktelow ARJ, Pearson RG. A systematic review of Vancouver B2 and B3 periprosthetic fractures. Bone Joint J. 2017;4:17-25.

19. Learmonth ID. The management of periprosthetic fractures around the femoral stem. J Bone Joint Surg Br. 2004;8:613-9.

20. Berry DJ. Periprosthetic fractures associated with osteolysis: a problem on the rise. J Arthroplasty. 2003;18:107-11.

Cite this article as: Mahi G, Lokhande MA, Yogi LK, Gawri A. A study of periprosthetic fractures following hemi-arthroplasty of hip in elderly population: diagnosis and management. Int J Res Orthop 2020;6:902-6. 